\title{
Meshless simulation of dam break using MLPG- RBF and shallow water equations
}

\author{
Juraj Mužík ${ }^{1, *}$, Martina Holičková ${ }^{1}$ \\ ${ }^{1}$ University of Zilina, Faculty of Civil Engineering - Department of geotechnics, Univerzitná 8215/1, \\ Slovakia
}

\begin{abstract}
This article focuses on the application of the meshless local Petrov-Galerkin (MLPG) method to solve the shallow water equations (SWE). This localized approach is based on the meshless weak formulation with the use of radial-basis functions (RBF) as the trial functions. Comparing with mesh-based methods, the present method is more efficient for large-scale problems with complex geometries. In this work, the numerical model is applied to simulate a dam-break problem as one of most descriptive benchmark problems for SWE. As a result, the adopted meshless method not only shows its algorithm applicability for class of problems described by SWE, but also brings more efficiency than several conventional mesh-based methods.
\end{abstract}

\section{Introduction}

The numerical models based on the shallow water equations (SWE) are commonly applied in hydraulic problems such as dam break analysis, open channel flows and sloshing wave problem.

Many numerical methods have been used to discretize numerical model described by the shallow water equations. For example, finite difference method (FDM) is very popular method that is simple to understand and code[1], finite volume method (FVM) has significant progress in simulation of flow phenomena[2] and also finite element method (FEM) has been successfully applied to the hydraulic simulation problems[3].

Due to the advantage of no mesh generation, the applications of meshless methods is current essential topics. In generally meshless methods have been classified into two main categories: boundary type and domain type. Under the premise that the fundamental or general solution of the governing partial differential equation (PDE) exists, boundary-type meshless methods have advantages from requiring relatively fewer computing nodes only around the boundary of the domain, such as the method of fundamental solutions (MFS) [4] and boundary knot method [5]. On the other hand, domain-type mesh-free methods have wider applications to the PDEs without fundamental solutions but need more nodes distributed in the whole domain. The numerical approximations of these methods are mainly based on the radial-basis functions (RBFs). For the SWE simulations, RBF strong form methods apply the interpolation of RBF to approximate the governing equations for

*Corresponding author: muzik@,fstav.uniza.sk 
examples multiquadrics (MQ) [6] or compactly supported radial-basis function (CSRBF) [7]).

The MLPG-RBF scheme has been developed by combining the RBF-based concept and the MLPG method. Atluri and his co-workers [8] initially introduced the MLPG method which followed the concept of weak form generalization. The conventional MLPG method has advantages of the high accurate solution, however the use of moving least-squares approximation (lack of delta property), brings some problems to the implementation of the boundary conditions. To overcome these difficulties the RBF interpolation method is adopted. Moreover, the band-width of the characteristic matrix can be adjusted using the concept of locally supporting nodes.

In this paper, we examine the applications of the MLPG-RBF to solve the SWE. First, the formulations will be introduced before the application of numerical procedures. The MLPG-RBF is introduced in chapter 3. In this chapter, the governing equations are discretized by the MLPG-RBF method in spatial domain and by Euler scheme in temporal direction. Chapter 4 demonstrates numerical example of the dam break. The model is verified by comparing the results with the benchmark solution in the literature.

Finally, conclusions and suggestions will be given based on the numerical model results.

\section{Two-dimensional shallow water equations}

The shallow water equations (SWEs, which are also called the de Saint-Venant equations) are used to describe flow behaviors in bodies of water where the horizontal length scales are much greater than the flow depth (i.e., the long wavelength phenomena). Here we briefly review the 2D SWEs.

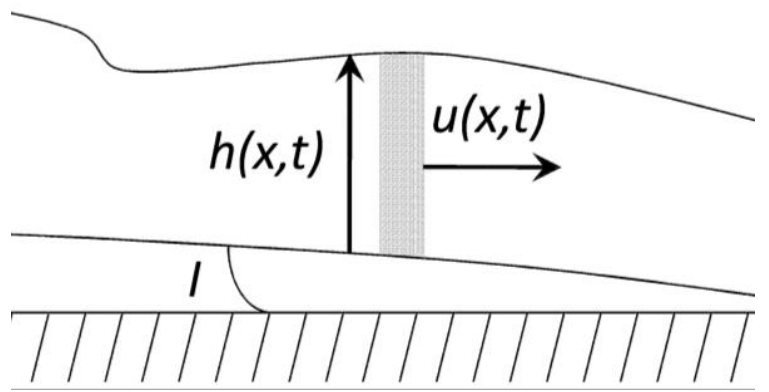

Fig. 1. Definition of the shallow-water flow region.

A shallow-water flow region is illustrated in Fig. 1. By the assumption of the hydrostatic pressure and uniform velocity profile along the vertical direction, the 3D incompressible Navier-Stokes equations can be simplified to the 2D SWEs.

In the primitive form, the governing equations including a continuity equation and a momentum equation in each of the $x$ - and $y$-directions are shown as follows:

$$
\frac{\partial h}{\partial t}+\frac{\partial(u h)}{\partial x}+\frac{\partial(v h)}{\partial y}=0
$$




$$
\begin{aligned}
& \frac{\partial(u h)}{\partial t}+\frac{\partial\left(u^{2} h\right)}{\partial x}+\frac{\partial(u v h)}{\partial y}+\frac{1}{2} g \frac{\partial h^{2}}{\partial x}=v_{e}\left(\frac{\partial\left(h u_{x}\right)}{\partial x}+\frac{\partial\left(h u_{y}\right)}{\partial y}\right)+F_{x} \\
& \frac{\partial(v h)}{\partial t}+\frac{\partial(u v h)}{\partial x}+\frac{\partial\left(v^{2} h\right)}{\partial y}+\frac{1}{2} g \frac{\partial h^{2}}{\partial y}=v_{e}\left(\frac{\partial\left(h v_{x}\right)}{\partial x}+\frac{\partial\left(h v_{y}\right)}{\partial y}\right)+F_{y}
\end{aligned}
$$

where $t$ is time, $h$ is the total water depth, $g=9.81 \mathrm{~m} / \mathrm{s}^{2}$ is the acceleration of gravity, $v_{e}$ is the kinematic eddy viscosity. $u$ and $v$ are depth-averaged velocity components in the horizontal $x$ - and $y$-directions, respectively. $u_{x}, u_{y}$ and $v_{x}, v_{y}$ are the derivatives of depthaveraged velocity components to $x$ - or $y$-direction according to its subscript. The forcing term used in our simulation contains only the bed-shear stress, which is given as

$$
F_{x}=\frac{g u \sqrt{u^{2}+v^{2}}}{C_{Z}^{2}} ; F_{y}=\frac{g v \sqrt{u^{2}+v^{2}}}{C_{Z}^{2}}
$$

where $C_{Z}=h^{(1 / 6)} / n$ is the Chezy friction coefficient and $n$ is the Manning coefficient. It is noted that the kinematic eddy viscosity $v_{e}$ of water is much smaller than the bed shear stress. Hence the viscous effect of kinematic eddy viscosity can be neglected in many hydrodynamics problems. By substituting continuity equation (1) into Eqs. (2) and (3) the momentum equations can be simplified as

$$
\begin{aligned}
& \frac{\partial u}{\partial t}+u \frac{\partial u}{\partial x}+v \frac{\partial u}{\partial y}+g \frac{\partial h}{\partial x}=\frac{F_{x}}{h} \\
& \frac{\partial v}{\partial t}+u \frac{\partial v}{\partial x}+v \frac{\partial v}{\partial y}+g \frac{\partial h}{\partial y}=\frac{F_{y}}{h}
\end{aligned}
$$

the former form (5) and (6) of governing equations has been used for the numerical model.

\section{The meshless local Petrov-Galerkin method with radial basis functions}

The MLPG method has been introduced by Atluri et al. [8]. It is characterized as meshless since distributed nodal points, covering the domain, are employed. All needed integrals (weak form) are carried out on the local subdomain centred at every point. All unknown variables are approximated by some interpolation method to obtain a system of non-linear equations. Solving this system of equations leads to a numerical solution of the problem. Atluri et al. [8] used the moving least squares (MLS) approximation scheme but nowadays the radial basis functions (RBFs) interpolation can be used instead (see e.g. [9]). An important advantage of RBF interpolation is an existence of the delta property and therefore the boundary conditions of the first kind can be easily defined.

\subsection{Methodology}


The entire domain $\Omega_{s}$ is covered by nodes located inside the area and also on the global boundary $\Gamma$ (see Fig. 2).

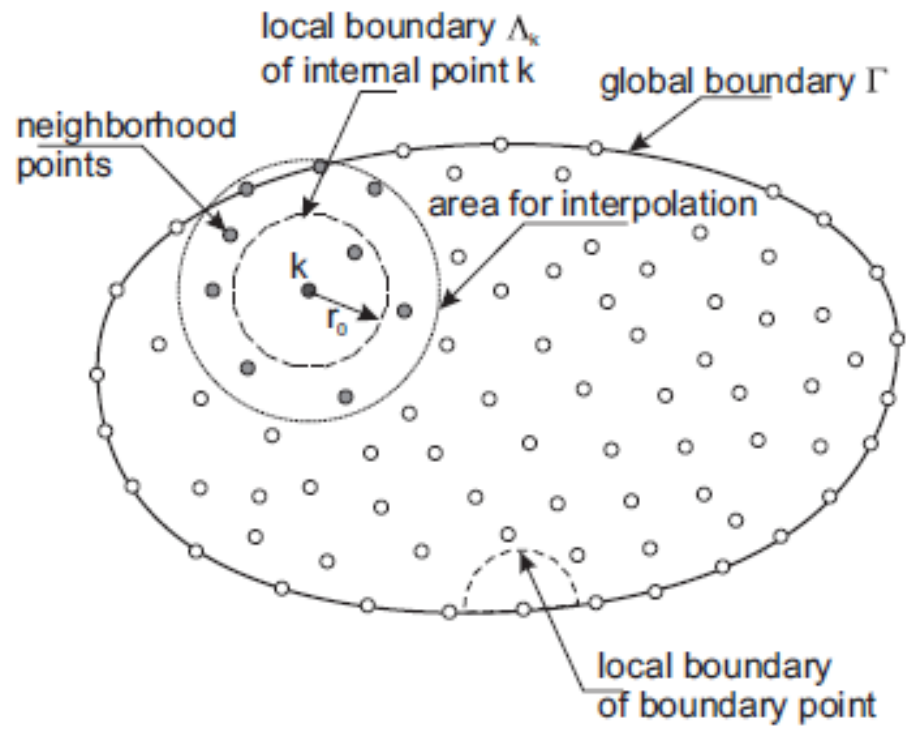

Fig. 2. Geometrical definition of the meshless model.

The mutual relationship of particular nodes is based on some interpolation algorithm. The local radial basis functions (RBFs) are used to approximate unknown height and velocities in the neighbourhood or support of a reference point $i$. Multiquadric functions are one of the most popular radial functions used for this purpose and they have been used in our paper. They can be defined as

$$
R\left(r_{i j}\right)=\sqrt{r_{i j}^{2}+c^{2}}
$$

where $r_{i j}$ is a distance between points $i$ and $j$ and $c$ is the so-called shape factor of multiquadric function. The formula of Hardy [10] with a slight modification is applied to the local RBFs (see [9]) to find the optimal value of the shape factor, which can be computed in point $i$ as

$$
c=\frac{0.815}{N} \sum_{j=1}^{N} r_{i j}
$$

The interpolation of the unknown variables can be written using the basis functions $\varphi_{i j}$ in the form (more details can be found in [9])

$$
h_{i}=\sum_{j=1}^{N} \varphi_{i j} h_{j}, \quad u_{i}=\sum_{j=1}^{N} \varphi_{i j} u_{j}, \quad v_{i}=\sum_{j=1}^{N} \varphi_{i j} v_{j}
$$

where $N$ is the number of neighbourhood points in the support domain. 


\subsection{The discrete form}

The five supporting points for point $i$ inside of computational domain $\Omega$ (including the point $i$ itself) were used for the unknown values interpolation at node $i$.

To obtain a local weak form of the shalow water flow in the reference point $i,(1),(5)$ and (6) is weighted by test functions $w_{i}$ and integrated over the local sub-domain $\Omega_{s}$ and the following equations are obtained

$$
\begin{aligned}
& \int_{\Omega_{S_{i}}} w_{i}\left[\frac{\partial h}{\partial t}+h \frac{\partial u}{\partial x}+u \frac{\partial h}{\partial x}+h \frac{\partial v}{\partial y}+v \frac{\partial h}{\partial y}\right] d \Omega_{S_{i}}=0 \\
& \int_{\Omega_{S_{i}}} w_{i}\left[\frac{\partial u}{\partial t}+u \frac{\partial u}{\partial x}+v \frac{\partial u}{\partial y}+g \frac{\partial h}{\partial x}\right] d \Omega_{S_{i}}=\int_{\Omega_{S_{i}}} w_{i}\left[\frac{F_{x}}{h}\right] d \Omega_{S_{i}} \\
& \int_{\Omega_{S_{i}}} w_{i}\left[\frac{\partial v}{\partial t}+u \frac{\partial v}{\partial x}+v \frac{\partial v}{\partial y}+g \frac{\partial h}{\partial y}\right] d \Omega_{S_{i}}=\int_{\Omega_{S_{i}}} w_{i}\left[\frac{F_{y}}{h}\right] d \Omega_{S_{i}}
\end{aligned}
$$

Spline weight functions $w_{i}$ with compact support have been considered in this paper

To compute integrals in (10), (11) and (12) we used a quadrilateral shape of the subdomain. The non-linear 2D SWEs consisting of Eqs. (10), (11) and (12) are discretized by the MLPG-RBF method in spatial domain and by full-implicit scheme (including all the external forcing terms) in temporal direction, and the discrete form, obtained using principles described in Chapter 3.1, at $i$ th node can be written as follows:

$$
\begin{aligned}
& \int_{\Omega_{S_{i}}} w_{i}\left[\frac{1}{\Delta t} \sum_{j=1}^{N} \varphi_{i j} h_{j}^{n+1}+\sum_{j=1}^{N} \varphi_{i j} \widetilde{v}_{j} \sum_{j=1}^{N} \frac{\partial \varphi_{i j}}{\partial y} h_{j}^{n+1}+\sum_{j=1}^{N} \varphi_{i j} \tilde{u}_{j} \sum_{j=1}^{N} \frac{\partial \varphi_{i j}}{\partial x} h_{j}^{n+1}\right] d \Omega_{S_{i}}+ \\
& \int_{\Omega_{S_{i}}} w_{i}\left[\sum_{j=1}^{N} \varphi_{i j} \tilde{h}_{j} \sum_{j=1}^{N} \frac{\partial \varphi_{i j}}{\partial x} u_{j}^{n+1}\right] d \Omega_{S_{i}}+\int_{\Omega_{S_{i}}} w_{i}\left[\sum_{j=1}^{N} \varphi_{i j} \tilde{h}_{j} \sum_{j=1}^{N} \frac{\partial \varphi_{i j}}{\partial y} v_{j}^{n+1}\right] d \Omega_{S_{i}}= \\
& \int_{\Omega_{S_{i}}} w_{i}\left[\frac{1}{\Delta t} \sum_{j=1}^{N} \varphi_{i j} h_{j}^{n}\right] d \Omega_{S_{i}} \\
& \int_{\Omega_{S_{i}}} w_{i}\left[\frac{1}{\Delta t} \sum_{j=1}^{N} \varphi_{i j} u_{j}^{n+1}+\sum_{j=1}^{N} \varphi_{i j} \tilde{v}_{j} \sum_{j=1}^{N} \frac{\partial \varphi_{i j}}{\partial y} u_{j}^{n+1}+\sum_{j=1}^{N} \varphi_{i j} \tilde{u}_{j} \sum_{j=1}^{N} \frac{\partial \varphi_{i j}}{\partial x} u_{j}^{n+1}\right] d \Omega_{S_{i}}+ \\
& \int_{\Omega_{S_{i}}} w_{i}\left[g \sum_{j=1}^{N} \frac{\partial \varphi_{i j}}{\partial x} h_{j}^{n+1}\right] d \Omega_{S_{i}}+\int_{\Omega_{S_{i}}} w_{i}\left[\frac{F_{x}}{h}\right] d \Omega_{S_{i}}=\int_{\Omega_{S_{i}}} w_{i}\left[\frac{1}{\Delta t} \sum_{j=1}^{N} \varphi_{i j} u_{j}^{n}\right] d \Omega_{S_{i}}
\end{aligned}
$$




$$
\begin{aligned}
& \int_{\Omega_{S_{i}}} w_{i}\left[\frac{1}{\Delta t} \sum_{j=1}^{N} \varphi_{i j} v_{j}^{n+1}+\sum_{j=1}^{N} \varphi_{i j} \widetilde{v}_{j} \sum_{j=1}^{N} \frac{\partial \varphi_{i j}}{\partial y} v_{j}^{n+1}+\sum_{j=1}^{N} \varphi_{i j} \tilde{u}_{j} \sum_{j=1}^{N} \frac{\partial \varphi_{i j}}{\partial x} v_{j}^{n+1}\right] d \Omega_{S_{i}}+ \\
& \int_{\Omega_{S_{i}}} w_{i}\left[g \sum_{j=1}^{N} \frac{\partial \varphi_{i j}}{\partial y} h_{j}^{n+1}\right] d \Omega_{S_{i}}+\int_{\Omega_{S_{i}}} w_{i}\left[\frac{F_{y}}{h}\right] d \Omega_{S_{i}}=\int_{\Omega_{S_{i}}} w_{i}\left[\frac{1}{\Delta t} \sum_{j=1}^{N} \varphi_{i j} v_{j}^{n}\right] d \Omega_{S_{i}}
\end{aligned}
$$

to solve non-linear system of equations the fixed value iteration procedure is adopted. The upper index in the equations (13), (14) and (15) represents the time step, the values marked with tilde are the values obtained from the last iteration (fixed values). The weight function used in the equations (10), (11) and (12) resp. (13), (14) and (15) is 4th order spline function, more details can be found in [9].

\section{Numerical results - 2D dam-break problem by non-linear SWE model}

This problem models a partial dam-break for a rapid opening of a sluice gate with a non-symmetric breach and its ability to simulate discontinuous flows. The computational domain is a $200 \mathrm{~m}$ by $200 \mathrm{~m}$ region. A dam is located in the middle of the domain with 10 $m$ thickness. The initial water depth is $10 \mathrm{~m}$ on one side and $5 \mathrm{~m}$ on the other side of the dividing wall. At time $t=0$, the dam fails, and the water is released through the $75 \mathrm{~m}$ wide non-symmetric breach, as shown in Fig. 3.

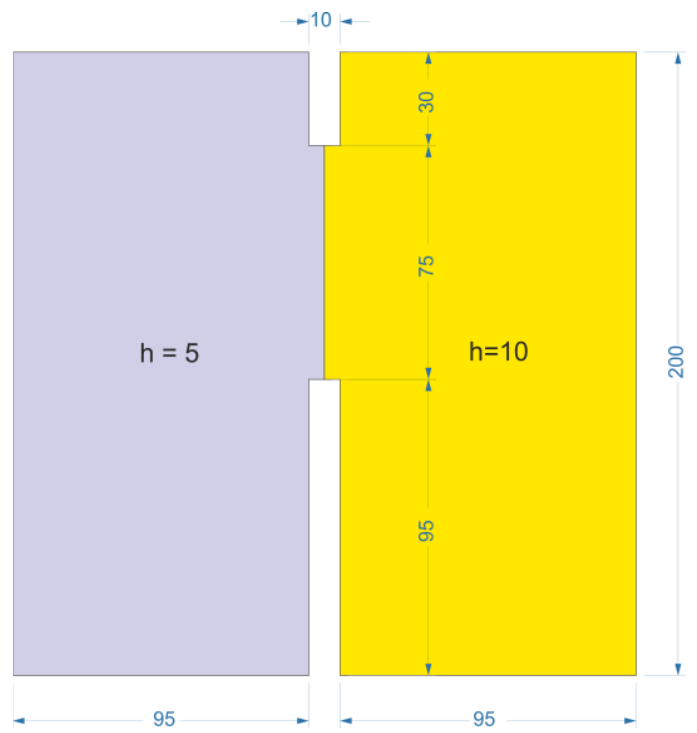

Fig. 3. Geometrical definition of the Dam-break model.

When the downstream water depth is $5 \mathrm{~m}$, the flow is subcritical everywhere. The boundary conditions at $x=0$ and $x=200 \mathrm{~m}$ are assumed to be transmissive and all other boundaries are considered as reflective. At the instant of the dam break, water is released through the breach, forming a positive wave propagating downstream and a negative wave spreading upstream. 


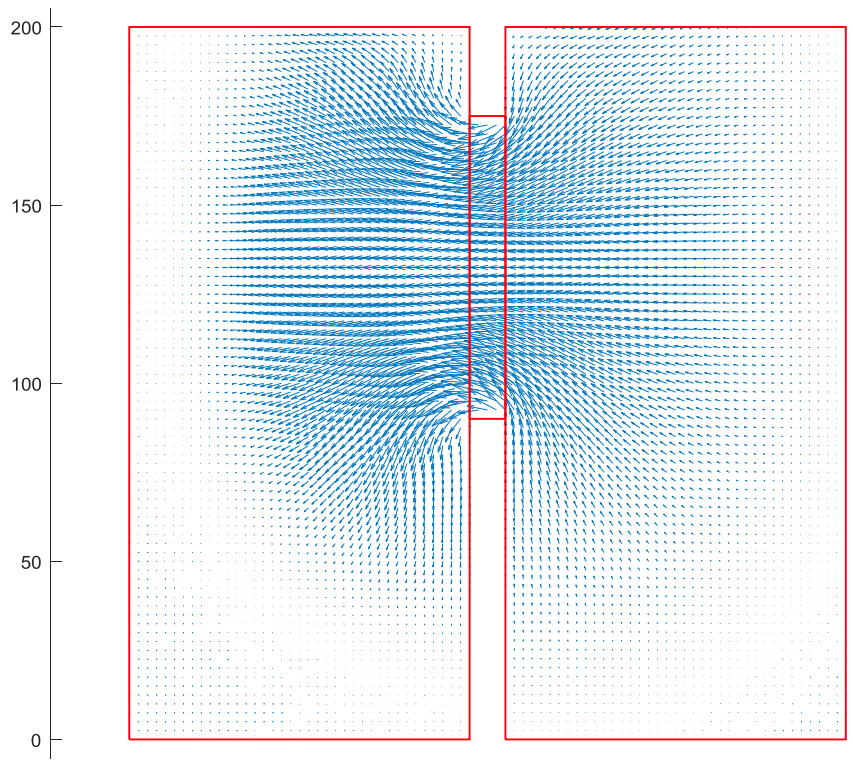

Fig. 4. Dam-break flow field vectors at $t=7.2 \mathrm{~s}$.

The scale of time step is given by the CFL condition as follows. At the beginning of the dam break caused by the gravity, we take the value of $u_{\max }$ by the velocity caused from the difference of the initial water elevation. By the initial water elevation, the main difference causing the dam break is on the $x$-direction and hence we also focus on the rule of $x$ direction. For $N=81 \times 81, \Delta x=200 / 80=2.5 \mathrm{~m}$ and by CFL condition $\Delta t$ needs to satisfy

$$
\Delta t \leq \frac{\Delta x}{u_{\max }}=\frac{2.5}{7} \approx 0.357 \mathrm{~s} .
$$

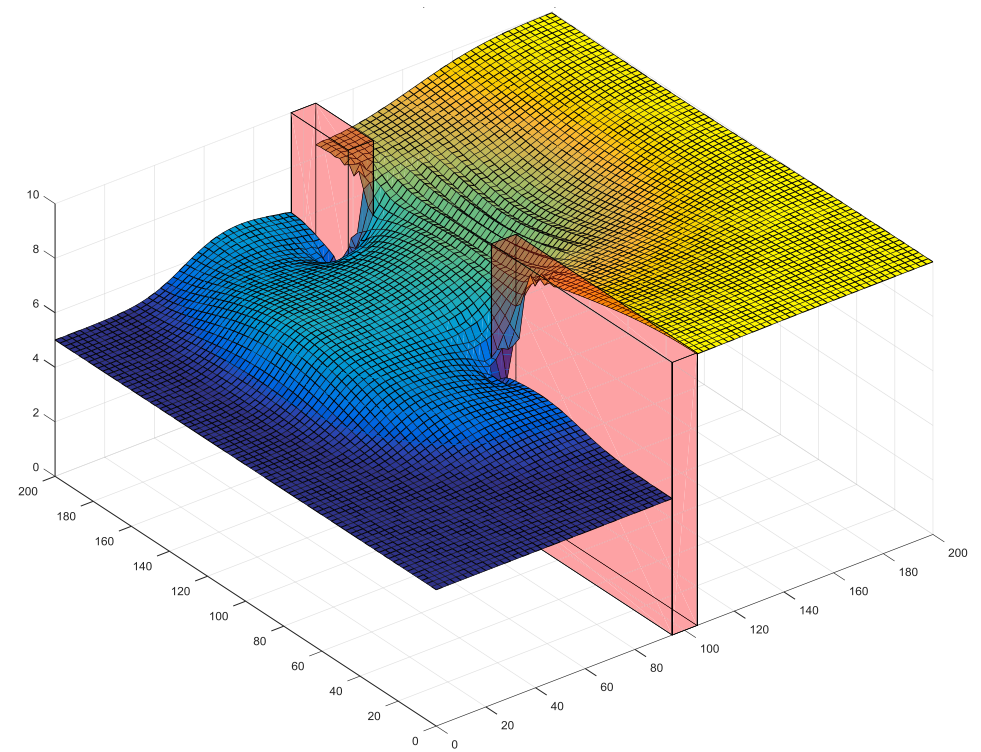

Fig. 5. Water surface profile of the 2-D dam-break problem at $t=7.2 \mathrm{~s}$. 
We compare our results by $N=81 \times 81$ at $t=7.2 s$ (see Fig.4, Fig.5), when the waves have not yet reached all the boundaries, with least-squares finite-element method (LSFEM) [11]. Other results of the form and propagating speed of the wave can be referenced in $[3,11]$. The left moving positive wave and right moving negative wave are both well resolved. The results were confirmed more stable to capture the fine details of the flow. The behaviour of the numerical scheme is in satisfactory agreement with computed results of these researches.

\section{Conclusions}

In this study, the applied local meshless method - the local meshless Petrov-Galerkin radial-basis-function (RBF) method is successfully applied to establish 2D shallow-water models for real engineering problems. The MLPG-RBF based 2D shallow-water models are verified by solving the dam-break problem compared with results of LSFEM. Results show good agreement with the results reported by other researches.

It is convinced that the proposed model not only provides accurate result but also has applicability to do inverse numerical analysis in real engineering project problem. In the further work, we plan to apply our SWEs to simulate more challenging flow problems, especially debris flow phenomena.

This contribution is the result of the project implementation: "Formulation of the new progressive numerical approaches for debris flow simulation" No. 1/0716/17 supported by the Scientific Grant Agency of Slovak Republic (VEGA).

\section{References}

1. J.C. Galland, N. Goutal, J.M. Hervouet, Advances in Water Resources, 14, 138, (1991)

2. E.F. Toro, Shock-capturing methods for free-surface shallow flows. (John Wiley and Sons, NewYork, 2001)

3. D.L. Young, Journal of the Chinese Institute of Engineers, 14, 143, (1991)

4. J. Xin-rong, W. Chen, Chinese Journal of Computational Mechanics, 28, 338, (2011)

5. W. Chen, M. Tanaka, Computers and Mathematics with Applications, 43, 379-391, (2002)

6. E.J. Kansa, Computers and Mathematics with Application, 19, 127-145, (1990)

7. X. Zhou, Y.C. Hon, K.F. Cheung, Engineering Analysis with Boundary Elements, 28, 967-973, (2004)

8. S.N. Atluri, T. Zhu, Computational Mechanics, 22, 117-127, (1998)

9. K. Kováŕík, J. Mužík, Engineering Analysis with Boundary Elements, 37, 187-196, (2013)

10. R.L. Hardy, Journal of Geophysical Research. 76, 1905-1915, (1971)

11. S.J. Liang, J.H. Tang, M.S. Wu, Acta Mechanica Sinica, 224, 523, (2008) 